\title{
Note on a thermal law of the wall for separating and recirculating flows
}

\author{
Daniel O.A. Cruz ${ }^{\text {a }}$, Atila P. Silva Freire ${ }^{b}$ \\ a Mechanical Engineering Department, CT/UFPA, Campus Universitário do Guamá, Federal University of Pará, \\ 66075-970, Belém, PA, Brazil \\ ${ }^{\mathrm{b}}$ Mechanical Engineering Program (PEM/COPPE/UFRJ), Federal University of Rio de Janeiro, C.P. 68503, \\ 21945-970 Rio de Janeiro, Brazil
}

Received 27 December 2000; received in revised form 6 July 2001

\begin{abstract}
The characteristics of the turbulent boundary layer near a separation point are studied here. The emphasis is on providing analytical expressions for the velocity and temperature near-wall solutions which are also valid in the region of reverse flow. These expressions can then be used as boundary conditions in numerical schemes that use two-equation differential models. The paper proposes a new expression for the description of the near-wall characteristic length. The temperature profiles near the wall are described by a newly proposed expression that reduces to a logarithmic profile in the attached region, and assumes a minus half power law profile at the separation point. (c) 2002 Elsevier Science Ltd. All rights reserved.
\end{abstract}

\section{Introduction}

The correct characterisation of a turbulent flow in the vicinity of a separation point and in the following recirculation region is a problem of considerable technological interest. A major difficulty in the analysis of the velocity and temperature fields near a separation point results from the vanishing of the shear stresses at the wall. This makes the classical asymptotic structure of the turbulent boundary layer not valid anymore since the main scaling parameters are reduced to zero. The clear implication is that alternative theories that resort to alternative scaling parameters have to be developed. Important examples are the works of Melnik [1], of Durbin and Belcher [2] and of Gersten [3]. All these works, however, are restricted to analyses of the velocity field only.

In a previous work, Cruz and Silva Freire [4] proposed a new asymptotic structure for the flow near a separation point, for both, the velocity and the temperature fields. In this work, a new scaling procedure

E-mail addresses: doac@ufpa.br (D.O.A. Cruz), atila@, serv.com.ufrj.br (A.P. Silva Freire). was developed which resulted in a changeable asymptotic structure for the boundary layer, different from those of other authors, but consistent with the experimental data. The flow structure was determined through the single limit concept of Kaplun [5] together with his Ansatz about domains of validity. The theory, in particular, led to a new expression for the velocity law of the wall and to a skin-friction equation that were supposed to hold up to the separation point and in the reverse flow region. Also, new expressions were proposed for the temperature law of the wall and for the Stanton number equation. All theoretical results were validated with the data of Vogel [6].

The purpose of the present work is to show how the theory of Cruz and Silva Freire [4] can be improved so as to furnish more reliable expressions for the velocity and temperature laws of the wall. In relation to the original paper, the following modifications are introduced here: (1) a new formulation for the reference velocity, $u_{\mathrm{R}}$, (2) a new expression for the velocity law of the wall, and (3) a new expression for the temperature law of the wall. The new reference velocity is specified through the total shear stress, as opposed to the previous one which had to be evaluated from an algebraic transcendental equation. The single expression 


\begin{tabular}{|c|c|c|c|}
\hline \multicolumn{2}{|c|}{ Nomenclature } & $u_{\mathrm{R}}$ & reference velocity \\
\hline$A$ & parameter in thermal law of the wall & \multirow{2}{*}{\multicolumn{2}{|c|}{ Greek symbols }} \\
\hline$A J$ & linear coefficient of thermal law of the wall & & \\
\hline$C_{\mathrm{f}}$ & skin friction coefficient & $x$ & von Karman's constant \\
\hline$c_{\mathrm{p}}$ & specific heat at constant pressure & & $(=0.4)$ \\
\hline$C_{\mu}$ & constant in $\kappa-\varepsilon$ model $(=0.09)$ & $\kappa$ & turbulent kinetic energy \\
\hline$E$ & parameter in law of the wall $(=9.8)$ & $\mu$ & viscosity \\
\hline$L$ & characteristic length & $v$ & kinematic viscosity \\
\hline $\operatorname{Pr}$ & Prandtl number & $\rho$ & density \\
\hline$P r_{\mathrm{t}}$ & turbulent Prandtl number & $\tau$ & shear stress \\
\hline$P$ & pressure & $\varepsilon$ & kinetic energy dissipation \\
\hline$Q$ & heat flux & \multicolumn{2}{|c|}{ Subscripts } \\
\hline$R$ & Reynolds number & $\mathrm{R}$ & reference parameter \\
\hline St & Stanton number & $\mathrm{p}$ & first grid point \\
\hline$T$ & temperature & $\mathrm{t}$ & temperature \\
\hline$u$ & longitudinal velocity component & $\mathrm{w}$ & conditions at wall \\
\hline$u_{\tau}$ & friction velocity & $\infty$ & external flow conditions \\
\hline
\end{tabular}

advanced here for the velocity law of the wall replaces the three expressions of Cruz and Silva Freire [4, Eqs. (25)-(27)]; this expression is supposed to hold in the whole fluid region. The temperature law of the wall is written with the help of reasonably sophisticated expressions for its angular and linear coefficients; these are a function of the turbulent Prandlt number, the pressure gradient, the reference velocity and the shear stress at the wall.

The main consequence of all these modifications is that much better results are found for the prediction of Stanton number near the separation point. This was a particular concern of the present authors when this work started.

Thus, the new formulation includes an alternative expression for the description of the near-wall characteristic length which holds also in the reverse flow region. The temperature field near the wall is described by a single expression that reduces to the logarithmic profile in the attached region, and assumes a minus half power law profile at the separation point. In the separated region the logarithmic profile is recovered.

The present formulation was thought as being very convenient for the implementation in turbulence models that resort to wall functions for the specification of wall boundary conditions (see $[4,7,8]$ ). In fact, the use of near-wall analytical solutions as boundary conditions in a numerical computation of the flow field is a practical means of avoiding all the complexities of the sublayer region.

Normally, flows which include separated regions are considered too complex for the specification of analytical local equilibrium boundary conditions. The practice then is to extend any specific turbulence model to the wall so that the viscous effects can be correctly captured. This gives origin to the low Reynolds number models [9]. Another approach that dispenses a particular treatment of the wall conditions is the second moment closure of Launder and Shima [10].

The present work has, therefore, striven in refining the classical law of the wall so that analytical near-wall solutions can still be used as boundary conditions in separated flows. The two proposed laws of the wall for the temperature and the velocity profiles will be applied to a $\kappa-\varepsilon$ turbulence model. The governing equations are discretised using a finite volume formulation coupled with an hybrid scheme for the treatment of the convective and diffusive terms simultaneously. The set of finite difference equations is solved using a very robust and intensively validated version of Teach Elliptic Axi-symmetrical Characteristics Heuristically (TEACH-2E) code which incorporates the SIMPLE algorithm specific for pressure velocity handling in incompressible flow. The test case adopted is the backward facing step experiments of Vogel [6]. A comparison with the classical formulation shows a great improvement in the calculation of the Stanton number.

\section{Velocity law of the wall}

Because the main concern of this work is to provide the means for a good numerical simulation of the flow near a separation point, the analytical results will be presented in a simplified fashion. For a complete description of the asymptotic solution of the problem, the reader is referred to Cruz and Silva Freire [4]. 
Following a procedure somewhat similar to the one derived by Cruz and Silva Freire [4], the law of the wall for a separating flow can be written as

$u=\frac{\tau_{\mathrm{w}}}{\left|\tau_{\mathrm{w}}\right|} \frac{2}{x} \sqrt{\frac{\tau_{\mathrm{w}}}{\rho}+\frac{1}{\rho} \frac{\mathrm{d} P_{\mathrm{w}}}{\mathrm{d} x} y}+\frac{\tau_{\mathrm{w}}}{\left|\tau_{\mathrm{w}}\right|} \frac{u_{\tau}}{\chi} \ln \left(\frac{y}{L_{\mathrm{c}}}\right)$,

where

$L_{\mathrm{c}}=\frac{\sqrt{\left(\frac{\tau_{\mathrm{w}}}{\rho}\right)^{2}+2 \frac{v}{\rho} \frac{\mathrm{d} P_{\mathrm{w}}}{\mathrm{d} x} u_{\mathrm{R}}}-\frac{\tau_{\mathrm{W}}}{\rho}}{\frac{1}{\rho} \frac{\mathrm{d} P_{\mathrm{W}}}{\mathrm{d} x}}$

and all symbols have their classical meaning; $\chi$ is the von Kármán constant $(=0.4), u_{\tau}$ is the friction velocity, and $u_{\mathrm{R}}(=\sqrt{\tau / \rho}, \tau=$ total shear stress $)$ is a reference velocity (which will be fully defined in the following through Eqs. (6) and (7)).

Eq. (1) is a generalisation of the classical law of the wall and replaces the three expressions advanced in $[4$, Eqs. (25)-(27)]. Eq. (2) is an expression for the nearwall region characteristic length, which is assumed to be valid in the attached and in the reverse flow regions. Far away from the separation point, where the shear stress is positive and $y\left(\mathrm{~d} P_{\mathrm{w}} / \mathrm{d} x\right) \ll \tau_{\mathrm{w}}$, Eq. (1) reduces to

$u=\frac{2}{x} u_{\tau}+\frac{u_{\tau}}{x} \ln \left(\frac{y}{L_{\mathrm{c}}}\right), \quad L_{\mathrm{c}}=v / u_{\tau}$,

that is, to the classical law of the wall.

Close to the separation point where $\tau_{\mathrm{w}}=0$, Eq. (1) reduces to

$u=\frac{2}{x} \sqrt{\frac{y}{\rho} \frac{\mathrm{d} P_{\mathrm{w}}}{\mathrm{d} x}}$,

an equation similar to Stratford's equation (see [11]).

In the reverse flow region where $y\left(\mathrm{~d} P_{\mathrm{w}} / \mathrm{d} x\right) \gg \tau_{\mathrm{w}}$, Eq.

(1) can be written as

$u=-\frac{2}{x} u_{\tau}-\frac{u_{\tau}}{x} \ln \left(\frac{y}{L_{\mathrm{c}}}\right), \quad L_{\mathrm{c}}=2\left|\frac{\tau_{\mathrm{w}}}{\mathrm{d} P_{\mathrm{w}} / \mathrm{d} x}\right|$.

Some comments seem now in order. The form of Eq. (1) was entirely inspired by Eqs. (25)-(27) and (36) of Cruz and Silva Freire [4]. In fact, the equations remain the same but for a major simplification achieved by changing the arguments of the logarithmic terms by $y / L_{\mathrm{c}}$. The reference length $L_{\mathrm{c}}$ defined by Eq. (2) is not new; in Cruz and Silva Freire [4] it had been previously introduced through Eq. (36). The generalisation provided by Eq. (1), however, implied that the friction velocity, $u_{\tau}$, used in the definition of $L_{\mathrm{c}}$ had to be replaced by the reference velocity $u_{\mathrm{R}}$. Finally, note that the characteristic length in the reverse flow region is different from the classical characteristic length given by Eq. (3). Eq. (5) is in agreement with Simpson et al. [12] which suggested that a characteristic length for the backflow region should be directly proportional to the absolute value of the wallshear stress.

We will now describe how the wall-shear stress can be evaluated from the above equations and through the use of a $\kappa-\varepsilon$ model.

A clear difficulty with the implementation of Eq. (1) as a boundary condition in a numerical code is that the wall-shear stress cannot be obtained in an explicit form. The numerical solution of Eq. (1) for the wall-shear stress is not a stable process which can therefore affect code robustness. To avoid these problems a linearisation procedure for Eq. (1) was developed.

The total shear stress can be evaluated from

$\tau_{\mathrm{p}}=C_{\mu}^{1 / 2} \rho \kappa_{\mathrm{p}}+\mu\left|\frac{\partial u}{\partial y}\right|_{\mathrm{p}}$

where the subscript $p$ denotes the first grid point.

The reference velocity $u_{\mathrm{R}}$ can then be directly determined from

$u_{\mathrm{R}}=\sqrt{\frac{\tau_{\mathrm{p}}}{\rho}}$.

Eq. (6) was obtained from a momentum balance in the near-wall region; it is similar to a relation usually employed by other authors to relate the wall-shear stress to the turbulent kinetic energy in a $\kappa-\varepsilon$ formulation (see, e.g., [13]), the only difference here is the inclusion of the viscous term to improve calculations when the first node near the wall is located at a distance shorter than $y / L_{\mathrm{c}} \leqslant 30$.

This equation can be used as a first estimate for the wall-shear stress if we consider

$\tau_{\mathrm{wo}}=\frac{u_{\mathrm{p}} C_{v}^{1 / 4} \tau_{\mathrm{p}}^{1 / 2} \rho^{1 / 2} \chi}{\ln \left(E y \frac{\left(\tau_{\mathrm{p}} / \rho\right)^{1 / 2}}{v}\right)}$.

with $E=9.8$.

In order to maintain code stability, the pressure gradient at the wall was obtained using Eqs. (6) and (8) to furnish the following equation:

$\frac{\mathrm{d} P_{\mathrm{w}}}{\mathrm{d} x}=\frac{\tau_{\mathrm{p}}-\tau_{\mathrm{wo}}}{y_{\mathrm{p}}}$.

This equation was obtained directly from the inner layer approximated equations; it represents the balance of forces in that layer.

Next, the characteristic length can be calculated from

$L_{\mathrm{c}}=\frac{\sqrt{\left(\frac{\tau_{\mathrm{wo}}}{\rho}\right)^{2}+2 \frac{v}{\rho} \frac{\mathrm{d} P_{\mathrm{w}}}{\mathrm{d} x} u_{\mathrm{R}}}-\frac{\tau_{\mathrm{wo}}}{\rho}}{\frac{1}{\rho} \frac{\mathrm{d} P_{\mathrm{w}}}{\mathrm{d} x}}$. 
Finally, the wall-shear stress is calculated from

$$
\tau_{\mathrm{w}}=\frac{u_{\mathrm{p}} \tau_{\mathrm{p}}^{1 / 2} \rho^{1 / 2} \chi}{2 \sqrt{\left|\frac{\tau_{\mathrm{p}}}{\tau_{\mathrm{w}}}\right|}+\ln \left(\frac{y_{\mathrm{p}}}{L_{\mathrm{c}}}\right)} .
$$

Using some production-dissipation equilibrium assumptions and Eq. (1) the kinetic energy dissipation and the production terms can be cast, respectively, as follows:

$$
\begin{aligned}
\varepsilon=C_{\mu}^{1 / 2} \kappa_{\mathrm{p}}( & \left.\frac{\left(\tau_{\mathrm{p}} / \rho\right)^{1 / 2}}{x y}+\frac{\frac{1}{\rho} \frac{\mathrm{d} P_{\mathrm{w}}}{\mathrm{d} x}}{x\left(\tau_{\mathrm{p}} / \rho\right)^{1 / 2}}\right), \\
\text { Production }= & \frac{C_{\mu}^{1 / 2} \kappa_{\mathrm{p}} \rho}{y}\left(\frac{2\left(\tau_{\mathrm{p}} / \rho\right)^{1 / 2}}{x}\right. \\
& \left.+\frac{\left|\left(\tau_{\mathrm{wo}} / \rho\right)\right|^{1 / 2}}{x} \ln \left(\frac{y}{L_{\mathrm{c}}}\right)\right) .
\end{aligned}
$$

\section{Temperature law of the wall}

An asymptotic theory for the thermal boundary layer near a separation point is also described in some detail in [4]. Because the temperature profiles can be written in terms of reference parameters already determined for the velocity profiles, the three Eqs. (28)-(30) of Cruz and Silva Freire [4] can be re-written here in a simplified form. Thus, the temperature law of the law can be cast as

$$
\frac{T_{\mathrm{w}}-T}{Q_{\mathrm{w}}}=\frac{P_{\mathrm{rt}}}{\chi_{\mathrm{t}} \rho c_{\mathrm{p}} u_{\tau}} \ln \frac{\sqrt{\tau_{\mathrm{w}} / \rho+\frac{1}{\rho} \frac{\mathrm{d} P_{\mathrm{w}}}{\mathrm{dx}} y}-\sqrt{\tau_{\mathrm{w}} / \rho}}{\sqrt{\tau_{\mathrm{w}} / \rho+\frac{1}{\rho} \frac{\mathrm{d} P_{\mathrm{w}}}{\mathrm{d} x}}+\sqrt{\tau_{\mathrm{w}} / \rho}}+C_{q},
$$

where

$$
\begin{aligned}
& C_{q}=\frac{P_{\mathrm{rt}}}{\chi_{\mathrm{t}} \rho c_{\mathrm{p}} u_{\mathrm{R}}} \ln \frac{4 E u_{\mathrm{R}}^{3}}{v\left|\frac{\mathrm{d} P_{\mathrm{w}}}{\mathrm{d} x}\right|}+A J, \\
& A J=1.11 P_{\mathrm{rt}} \sqrt{\frac{A}{x}}\left(\frac{P_{\mathrm{r}}}{P_{\mathrm{rt}}}-1\right)\left(\frac{P_{\mathrm{r}}}{P_{\mathrm{rt}}}\right)^{0.25}, \\
& A=26 \frac{\left|\tau_{\mathrm{w}} / \rho\right|^{1 / 2}}{u_{\mathrm{R}}}, \quad P_{\mathrm{rt}}=0.9,
\end{aligned}
$$

and all symbols have their classical meaning.

To improve the performance of Eq. (14) its linear coefficient was replaced by a more sophisticated equation. $C_{q}$ was basically developed so that Eq. (14) reduces to the classical law of the wall far away for a separation point. Eq. (16) was first proposed by Launder and Spalding [12]. Eq. (17) has been modified from the original formulation $(A=26)$ in order to perform better in the separated flow region. In [4] the predicted values of $S_{\mathrm{t}}$ were well below the experimental values so that Eqs. (15)-(17) had to be introduced to rectify that.

\section{Results}

The results found with the present formulation will now be compared with the standard $\kappa-\varepsilon$ model and the data of Vogel [6] for the backward facing step flow. The flow conditions of Vogel are shown in Table 1.

The governing equations are discretised using a finite volume formulation coupled with an hybrid scheme for the treatment of the convective and diffusive terms simultaneously. The set of finite difference equations is solved using a very robust and intensively validated version of TEACH-2E code which incorporates the SIMPLE algorithm specific for pressure velocity handling in incompressible flow. The grid had $146 \times 102$ points. The computational domain is shown in Fig. 1.

To perform the calculations the limit of the viscous region was taken as $y^{+}=5$. For flows subjected to a zero pressure gradient, one normally considers this region to be defined by $y^{+}=11$. For the present experimental conditions, however, we found $y^{+}=5$ to be the appropriate value. Please note that we have taken the data from Vogel's doctoral thesis which are different from the data presented in [14]. The result is that the curves and experimental points to be shown here do not coincide with the corresponding ones in [4].

The next figures show the results for the computed velocity and temperature profiles, for several stations (see Figs. 2-4). All figures present three curves: one with the experimental data of Vogel [6], one with the present computations, and a final one with computations made with the standard $\kappa-\varepsilon$ model. The velocity

Table 1

The experimental data of Vogel [6]

\begin{tabular}{llll}
\hline Author & $U(\mathrm{~m} / \mathrm{s})$ & $R$ & $Q_{\mathrm{w}}\left(\mathrm{W} / \mathrm{m}^{2}\right)$ \\
\hline Vogel [6] & 11.3 & 28,000 & 270 \\
\hline
\end{tabular}

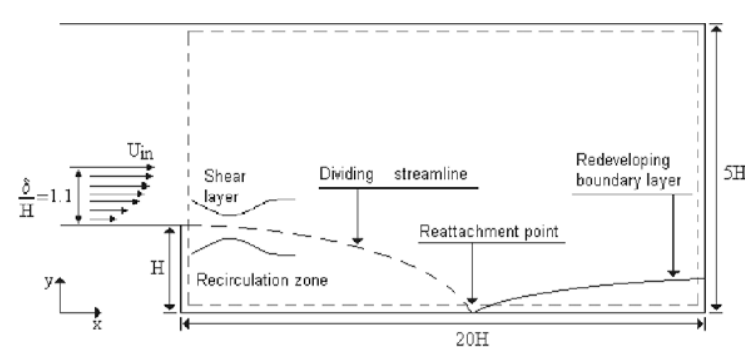

Fig. 1. Flow domain. 


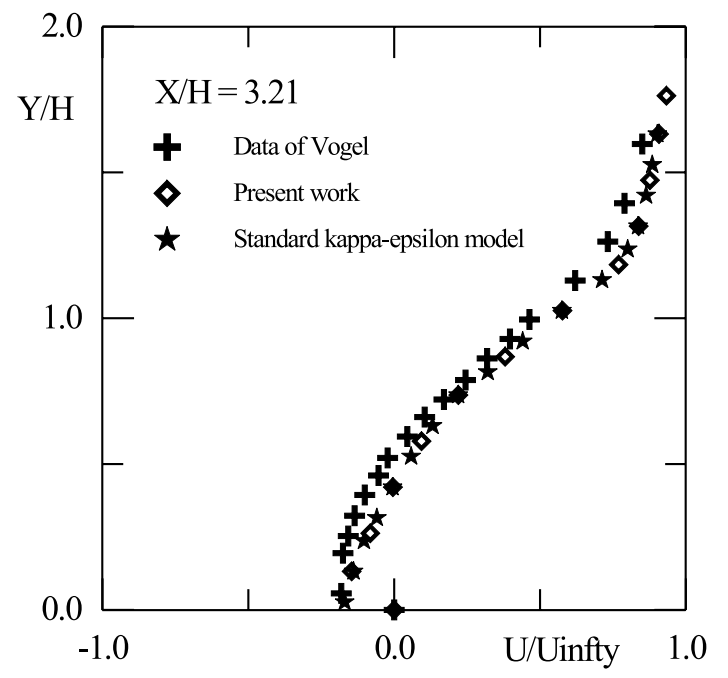

Fig. 2. Velocity profiles, $X / H=3.21$ : $(+)$, data of Vogel [6]; $(\diamond)$, present work; (灾), standard $\kappa-\varepsilon$ model.

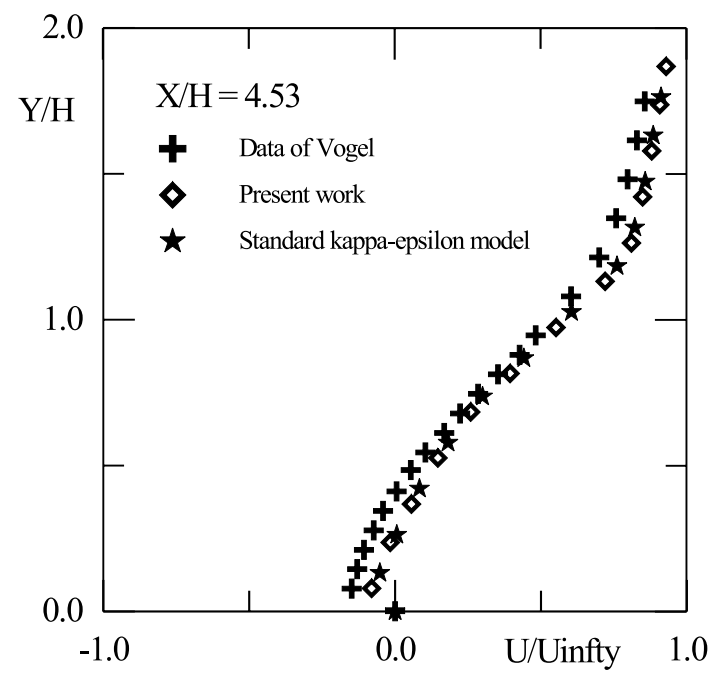

Fig. 3. Velocity profiles, $X / H=4.53$ : (+), data of Vogel [6]; $(\diamond)$, present work; (它), standard $\kappa-\varepsilon$ model.

profiles show that the present formulation only alters the results for the very near-wall region. Indeed, for most of the velocity profile, both numerical appproaches give very close data. Through the velocity profiles, the position of the point of flow reattachment can also be estimated. Here, our solution starts to depart from the classical $\kappa-\varepsilon$ solution. The results are shown in Table 2.

For the temperature profiles, the differences between the present approach and the results given by the classical $\kappa-\varepsilon$ model are significant at the wall. This can be

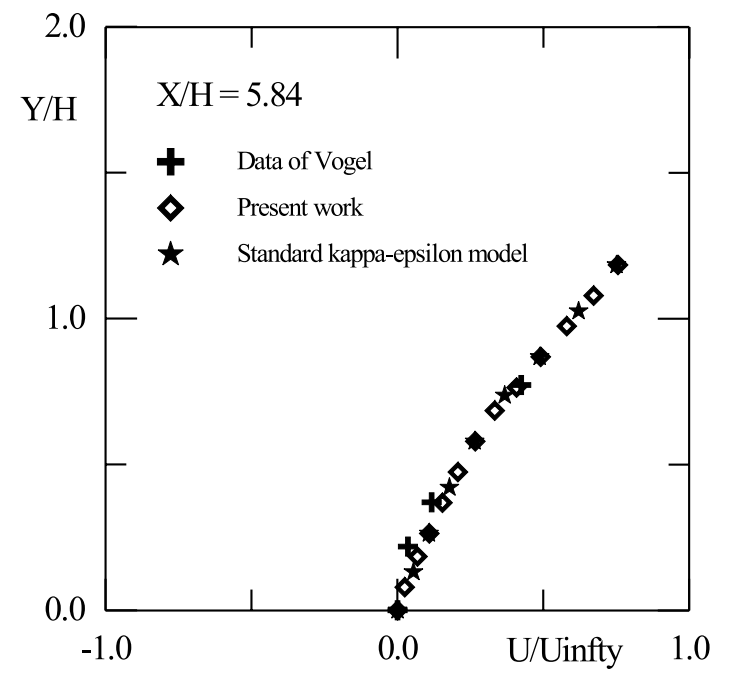

Fig. 4. Velocity profiles, $X / H=5.84$ : (+), data of Vogel [6]; $(\diamond)$, present work; $(\bowtie)$, standard $\kappa-\varepsilon$ model.

Table 2

Prediction of flow reattachment point

\begin{tabular}{ll}
\hline Reattachment point & $X / H$ \\
\hline Experiments & 6.6 \\
Present work & 6.0 \\
Standard $\kappa-\varepsilon$ model & 5.5 \\
\hline
\end{tabular}

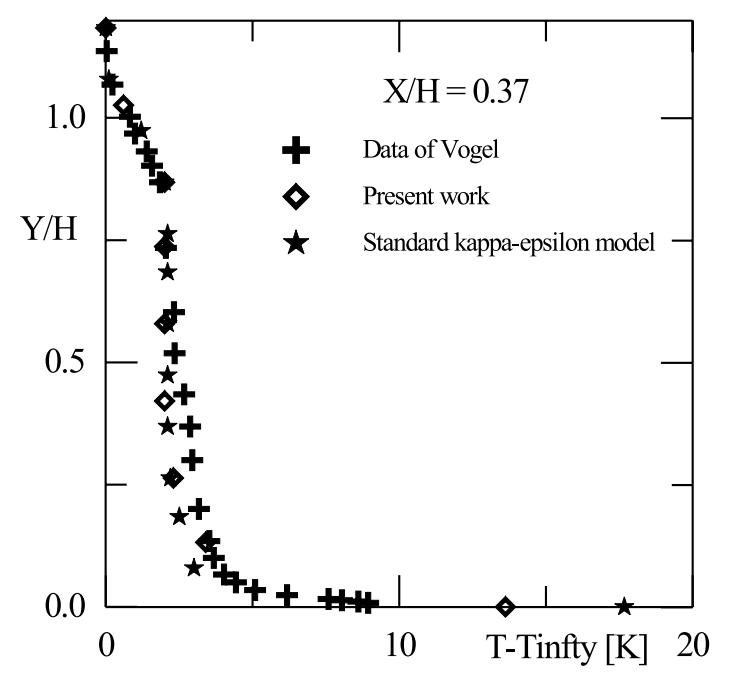

Fig. 5. Temperature profiles, $X / H=0.37$ : (+), data of Vogel [6]; $(\diamond)$, present work; $(\lesssim)$, standard $\kappa-\varepsilon$ model.

clearly seen from Figs. 5-9; note in particular that the first grid point presents very different values for both numerical predictions. Eq. (14) clearly provides results which are much closer to the experimental data than the 


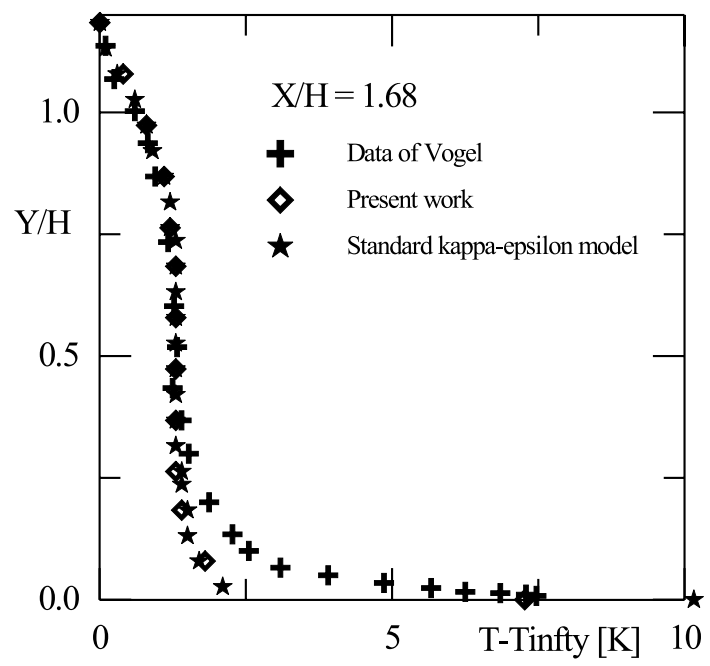

Fig. 6. Temperature profiles, $X / H=1.68:(+)$, data of Vogel [6]; $(\diamond)$, present work; (泳), standard $\kappa-\varepsilon$ model.

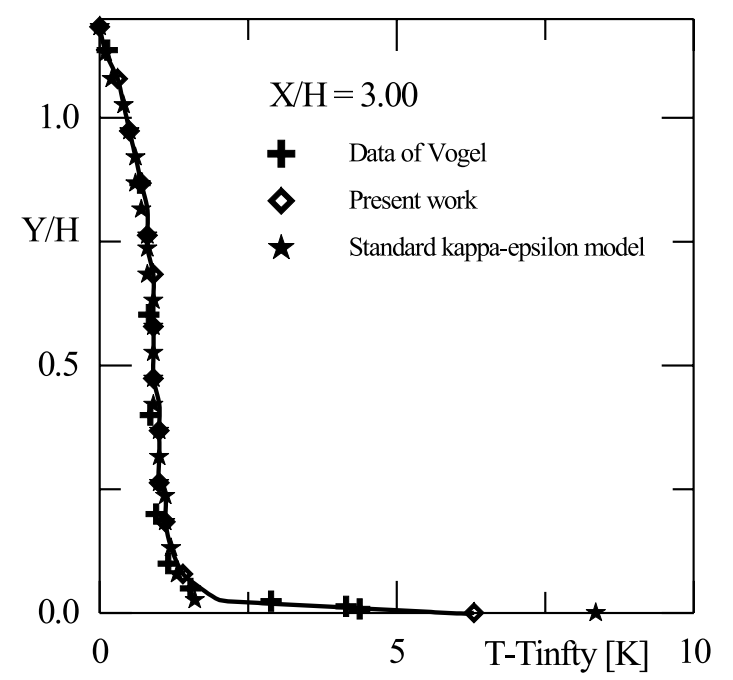

Fig. 7. Temperature profiles, $X / H=3.00:(+)$, data of Vogel [6]; $(\diamond)$, present work; (汭), standard $\kappa-\varepsilon$ model.

standard formulation and which will result in a much better prediction of the Stanton number. This fact is, indeed, directly connected with the estimation of Stanton number (see Fig. 10).

Results for the skin-friction coefficient and the Stanton number are shown next. The improvement in the predictions, thanks to the use of the new formulations for the law of the wall proposed here, is remarkable in the flow separation region. It is important to note that these results were obtained with no additional computational cost or loss of code robustness, in comparison with the original code which used the standard $\kappa-\varepsilon$ model.

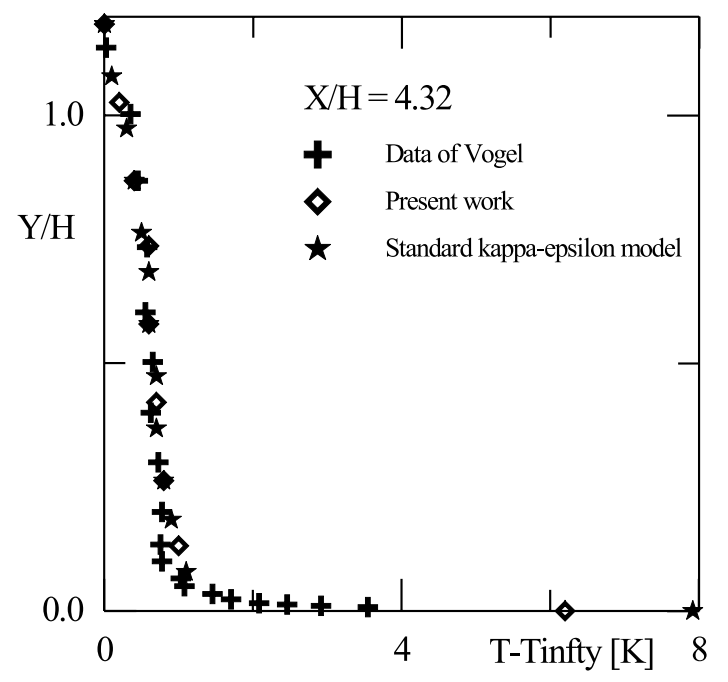

Fig. 8. Temperature profiles, $X / H=4.32:(+)$, data of Vogel [6]; $(\diamond)$, present work; (冘), standard $\kappa-\varepsilon$ model.

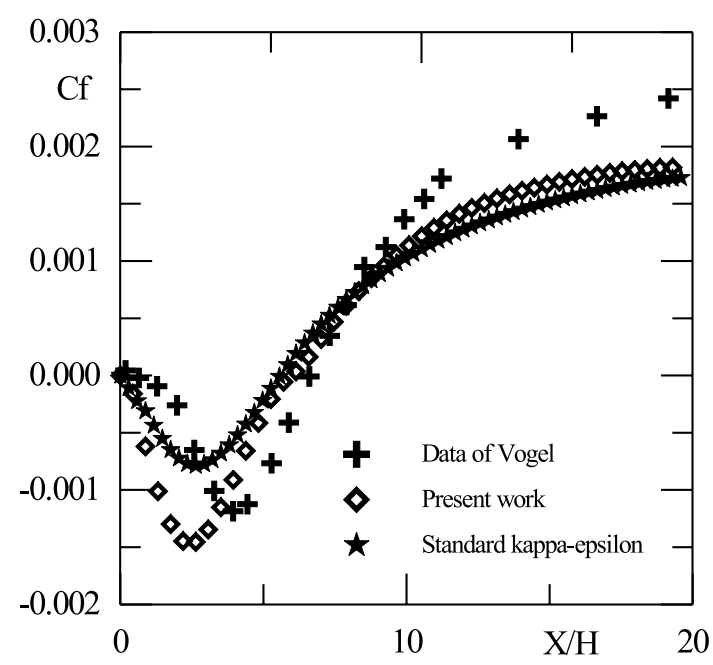

Fig. 9. Skin-friction results: $(+)$, data of Vogel [6]; $(\diamond)$, present work; (预), standard $(\kappa-\varepsilon)$ model.

\section{Conclusion}

The present work had a very distinct goal at its beginning, to provide an alternative method for the calculation of flows subjected to separation. Specially, we wanted to improve the calculation methods for the skinfriction coefficient and the Stanton number which were developed in the past to use the law of the wall.

Apparently, the goal has been achieved with the specification of expressions (1) and (14). These expressions were shown to stand very well against the data of Vogel [6], giving very good results for the velocity and 


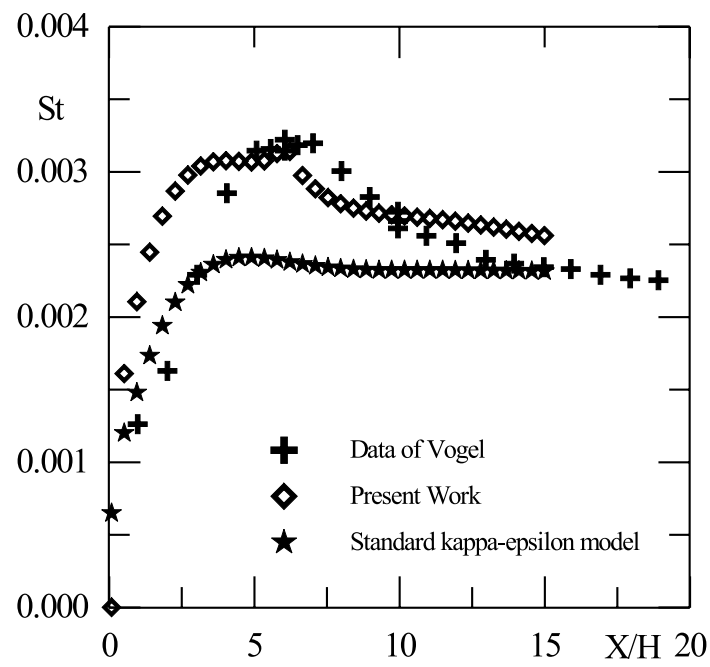

Fig. 10. Stanton number results: $(+)$, data of Vogel [6]; $(\diamond)$, present work; (冘), standard $\kappa-\varepsilon$ model.

temperature fields and the skin-friction coefficient and the Stanton number. We have chosen the data of Vogel [6] as our reference data for they represent the most detailed account of the problem we have so far encountered. Through his thesis we had access to a complete set of tabulated data which could be used in detail for validation of the present approach.

Of course, a limitation of the present simulation is its inability to capture any unsteadiness occurring in the flow. As such the location of the re-attachment point is fixed, so is the location of the point of zero $C_{\mathrm{f}}$ and maximum $S_{\mathrm{t}}$.

Presently, the authors are subjecting those expressions to further scrutiny. This will be reported in another occasion.

\section{Acknowledgements}

The present work was financially supported by the Brazilian National Research Council (CNPq) through grant No. 523476/96-5.

\section{References}

[1] R.E. Melnik, An asymptotic theory of turbulent separation, Compt. Fluids 17 (1989) 165-184.

[2] P.A. Durbin, S.E. Belcher, Scaling of adverse-pressuregradient turbulent boundary layers, J. Fluid Mech. 238 (1992) 699-722.

[3] K. Gersten, Some contributions to asymptotic theory for turbulent flows, in: 2nd International Symposium on Transport Phenomena in Turbulent Flows, Tokyo, Japan, 1987, pp. 201-214.

[4] D.O.A. Cruz, A.P. Silva Freire, On single limits and the asymptotic behaviour of separating turbulent boundary mayers, Int. J. Heat Mass Transfer 41 (1998) 2097-2111.

[5] S. Kaplun, Fluid Mechanics and Singular Perturbations, Academic Press, New York, 1967.

[6] J.C. Vogel, Heat transfer and fluid mechanics measurements in the turbulent reattaching flow behind a backwardfacing flow step, Ph.D. Dissertation, Stanford University, 1984.

[7] C.G. Chieng, B.E. Launder, On the calculation of turbulent transport downstream of an abrupt pipe expansion, Numer. Heat Transfer 3 (1980) 189-207.

[8] H. Ciofalo, M.W. Collins, $\kappa-\varepsilon$ predictions of heat transfer in turbulent recirculating flows using an improved wall treatment, Numer. Heat Transfer, Part B 15 (1989) 21-47.

[9] V.C. Patel, W. Rodi, G. Sheuerer, Turbulence models for near-wall and low-Reynolds number flows: a review, AIAA J. 23 (1985) 1308-1319.

[10] B.E. Launder, N. Shima, Second-moment closure for the near-wall sublayer: development and application, AIAA J. 10 (1989) 1319-1325.

[11] B.S. Stratford, An experimental flow with zero skin-friction throughout its region of pressure rise, J. Fluid Mech. 5 (1959).

[12] R.L. Simpson, Y.T. Chew, B.G. Shivaprasad, The structure of a separating turbulent boundary layer. Part 1: Mean flow and Reynolds stress, J. Fluid Mech. 113 (1981) 23-51.

[13] B.E. Launder, D.B. Spalding, The numerical computation of turbulent flows, Comp. Meth. Appl. Mech. 3 (1974) 269-289.

[14] J.C. Vogel, J.K. Eaton, Combined heat transfer and fluid dynamic measurements downstream of a backward facing step, J. Heat Transfer 107 (1985) 922-929. 